\title{
Studying Electrocatalyts in Operando Conditions: Correlating TEM Imaging and X-Ray Spectroscopies
}

Nathaly Ortiz Peña ${ }^{1}$, Dris Ihiawakrim ${ }^{1}$, David Portehault ${ }^{2}$, Christel Laberty-Robert ${ }^{2}$, Sophie Carenco $^{2}$, Clement Sanchez ${ }^{2}$, Ovidiu Ersen ${ }^{1}$

${ }^{1 .}$ Institut de Physique et Chimie des Matériaux de Strasbourg, 23 rue du Loess, BP 43, Strasbourg Cedex 2, France.

2. Sorbonne Université-UPMC Univ. Paris 6, CNRS, College de France, UMR 7574, 4 Place de Jussieu, 75005 Paris, France

Considering the enormous relevance of electrocatalysts in the search for more efficient, clean and affordable energy production methods [1], it is fundamental to understand their behavior in real conditions in order to be able to improve their performances in terms of activity and stability. However, the full comprehension of the dynamic processes in catalytic and electrocatalytic materials has been limited by the historical unsuitability of the highly resolved characterization techniques to track in real time and in operation conditions the morphological and electronic changes in the catalyst surface. Nowadays, thanks to the development of specialized holders, there is a blooming field of in situ and operando studies with different instruments allowing to move forward in the understanding of the real species playing a role in the catalytic mechanisms[2]. In this regard, herein we propose to combine complementary in situ analyses, based on TEM (local and morphological analysis) and synchrotron based X-ray spectroscopies (average and electronic analysis). This combination addresses intrinsic limitations on each side: the lack of averaging for TEM, and the absence of local information for X-ray spectroscopy.

From the in situ TEM front, since this type of correlative approach is quite recent and innovative; the first steps in this study were dedicated to stablish the adequate acquisition parameters in order to obtain results comparable with those from typical ex situ measurements and with other in situ techniques. As depicted in the figure 1, the observation is made through a thickness of electrolyte sandwiched between SiNx membranes were the working electrode is printed. Thus, we started by tackling some of the challenges inherent to the inclusion of flowing liquid and a dynamic stimulus: sample drift, electrochemical signal shift, radiolysis products, among others. In this sense, we have achieved to find a compromise between the imaging parameters like electron dose and exposure time, and the electrochemical parameter such as scan rate and electrolyte flow rate.

As a model system, we have chosen a relatively simple spinel structure of cobalt oxide, but of great importance in the field of water splitting devices and/or batteries [3]. The nanoparticles of $\mathrm{Co}_{3} \mathrm{O}_{4}$ were deposited over the electron transparent glassy carbon working electrode. The electroctalysis was then studied by cyclic voltammetry and chronoamperometry measurements. The results are comparable with those from the classical electrochemical set-up under the chosen observation parameters. In such way, we were able to track for the first time the changes in the catalyst nanoparticles. Although, these local changes have been typically described as completely reversible, our observations show that, in a basic electrolyte, an amorphization of the surface at the onset potential becomes irreversible. Moreover, this amorphization continues to expand in the new crystalline surface leading to a decrease in the crystallite size. Nonetheless, this surface expansive amorphization is not uniform in all the nanoparticles, fact that might be related to the exposed planes in each of them. The surface plane orientation is known to play an important role in the catalytic activity. Particularly, surfaces majorly form by $\mathrm{Co}_{3} \mathrm{O}_{4}$ (111) plane have shown to present the highest turnover efficiency, hence we think that the inhomogeneous amorphization rate could arise from a tendency of particles with (111) as surface planes to react first by forming amorphous species as $\mathrm{CoOOH}$ than then remain around the crystalline as a sort of catalytic matrix. 
The conclusions withdrawn from the in situ TEM studies will be confronted with the operando xray spectroscopic measurements performed at the synchrotron SOLEIL facilities. The correlation of these two approaches will allow to understand more clearly the real nature of the cobalt electronic state at the onset potential and the effect of the morphological or structural changes in the catalytic activity. This study is the first one on this type of system of high interest in electrocatalysis and more generally in the field of renewable energies. These results are very encouraging and stimulate the practical implementations of such in situ experiments since they clarify long-standing questions related to the behavior of the materials under operation.

\section{References:}

[1] ZW Seh et al, Science 355 (2017), eaad4998.

[2] J Dou et al, Chem Soc Rev 46 (2017), p. 2001.

[3] S Kalasina, N Phattharasupakun and M Sawangphruk, J Mater Chem A 6 (2017), p. 36.

[4] Z Chen, CX Kronawitter and BE Koel, Phys Chem Chem Phys 17 (2015), p. 29387.

[5] S Gupta et al, Nanoscale 10 (2018) p. 8806.

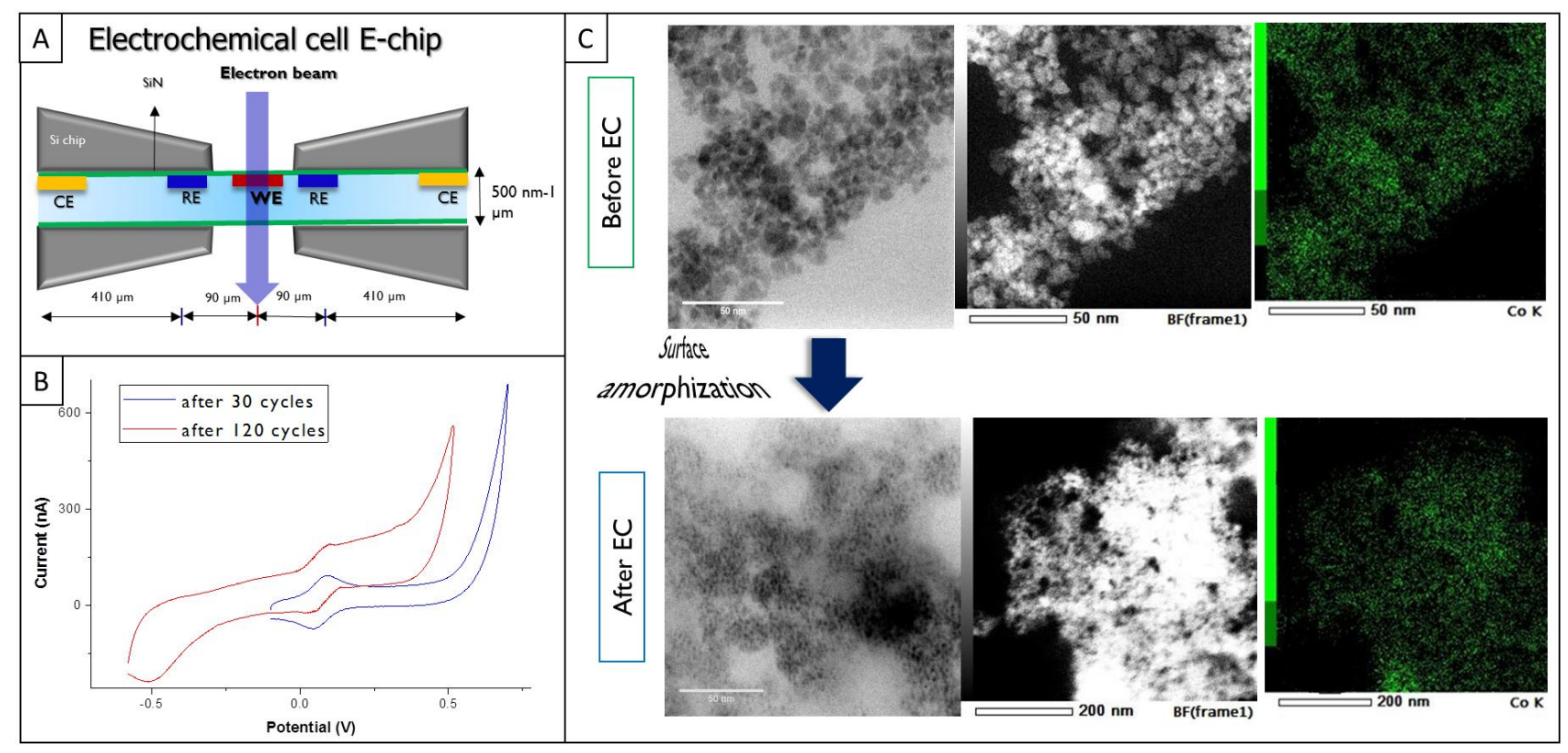

Figure 1. (A) Electrochemical E-chip use in the in situ TEM set-up. (B)Cyclic voltammograms of $\mathrm{Co}_{3} \mathrm{O}_{4}$ in OER conditions in situ after 30 cycles and after 120 cycles. (C) Pre and post-mortem STEM images and EDX mapping of the catalysts nanoparticles. 